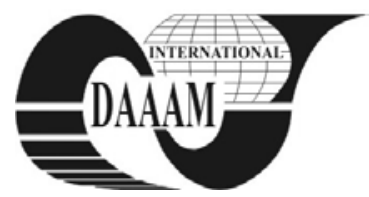

\title{
SOFTWARE FOR DEFORMITY AND STRESS CONDITION OF 2D TRUSS BEAMS ANALYSIS
}

\author{
MUMINOVIC, A[dis]; SARIC, I[sad] \& REPCIC, N[edzad]
}

\begin{abstract}
Modern commercial integrated CAD/CAM/CAE systems have a module for analysis of deformity and stress condition of structures, and it is based on Finite Element Method (FEM). Use of FEM method for structures analysis is conditioned by purchase of expensive CAD/CAM/CAE systems. This paper provides solutions that avoid need for purchase of these types of systems, thanks to the development of proper software for specific structures analysis. The possibilities of the developed software, named 2D-FEM, which is based on FEM method and intended for deformity and stress conditions analysis of the $2 D$ truss beams, are described in this paper. Software 2D-FEM is developed in C\# programming language, and its possibilities are demonstrated in the example of deformity and stress conditions of the selected $2 D$ truss beam. Key words: software, C\#, finite element method (FEM), 2D truss beams
\end{abstract}

\section{INTRODUCTION}

Using of FEM method for truss beams analysis becomes inevitable. A designer, on the base of a selected load, can obtain data about deformity and stress condition of truss beam, by its use. On the base of those data, a designer can notice so-called „critical” spots on truss beams, and after that, according to need, he/she can redesign it. In spite of that, the analysis of truss beams is almost impossible without a use of computer and modern commercial integrated CAD/CAM/CAE systems, thanks to mandatory and detailed estimate. These types of systems are very expensive, but their purchase can be avoided by the development of the proper software based on FEM method. The possibility of FEM method using for deformity and stress condition of $2 \mathrm{D}$ truss beams is discussed in the next paragraph.

\section{FEM METHOD USING FOR DEFORMITY AND STRESS CONDITION OF 2D TRUSS BEAMS ANALYSIS}

Application of FEM method for 2D truss beams analysis enables determination of displacements of all truss joints in direction of two axis of the selected coordinate system, for the selected load. Therefore, it is possible to notice deformity of beam under influence of the selected load. As it is known, elements of truss beam (members of truss beam) with different cross sections can be jointly or firmly tied. Developed 2D-FEM software, described in this paper, is intended for analysis of deformity and stress condition of 2D truss beams with jointly tied elements, in the case when momentum of bending are not considered in the estimate. The basic system of FEM method equations, which is used for estimation of displacements and external loads (forces), is:

$$
[K]\{\delta\}-\{R\}=0
$$

It presents system of equations of balance for truss beam (Budynas-Nisbett, 2006; Cook, 2002), where:

- $[K]$ - matrix which presents sum of widen matrixes of stiffness of elements,
- $\{\delta\}$ - displacements vector,

- $\{R\}$ - external loads vector.

Number of matrixes of elements stiffness corresponds to number of the beam elements. These matrixes are fixed by geometry and characteristics of the beam elements materials. Displacements vector represents a vector which encloses displacements of all truss joins in direction of axis. For the case of 2D truss beam every truss joint moves horizontaly and verticaly. Of course, this is in affect only in the case when the beam elements are jointly tied, and when bending moments are not taken into consideration. Displacements vector is in a following form:

$$
\{\delta\}=\left\{\begin{array}{c}
u_{11} \\
u_{21} \\
\vdots \\
u_{1 i} \\
u_{2 i}
\end{array}\right\}
$$

$i$ represents the number of the trusses joints, indexes 1 and 2 represent axis $x$ (horizontal displacement) and axis $y$ (vertical displacement). External loads vector enclose forces concentrated in the truss joints which influence the truss beam in direction of axis. External loads vector is in a following form:

$$
\{R\}=\left\{\begin{array}{c}
F_{11} \\
F_{21} \\
\vdots \\
F_{1 i} \\
F_{2 i}
\end{array}\right\}
$$

It is important to point out the fact that there is a large amounth of input data during manual calculations of these systems, therefore a large amount of data is obtained during the process of solving the selected problem. More over, one has to solve a large number of equations with the same number of unknowns during manual calculations. Therefore, there is a need for software to solve the problem.

\section{2D-FEM - SOFTWARE FOR DEFORMITY AND STRESS CONDITION OF 2D TRUSS BEAMS ANALYSIS}

\subsection{Phases of 2D-FEM Software Development}

2D-FEM software development designed for deformity and stress condition of $2 \mathrm{D}$ truss beams has gone through the following phases:

- problem identification

- determination of goal

- program design

- implementation

- testing

In fact, software 2D-FEM is the upgrade of the original console orientated software which is designed in 1985 in FORTRAN programming language. There was no graphic interface in original software, so there was no possibility for graphical presentation of the obtained results, or more exactly 
the truss beam in undeformed and deformed condition (Picuga, 1985). Algorithms for system solving (1) are used from the original console orientated software.

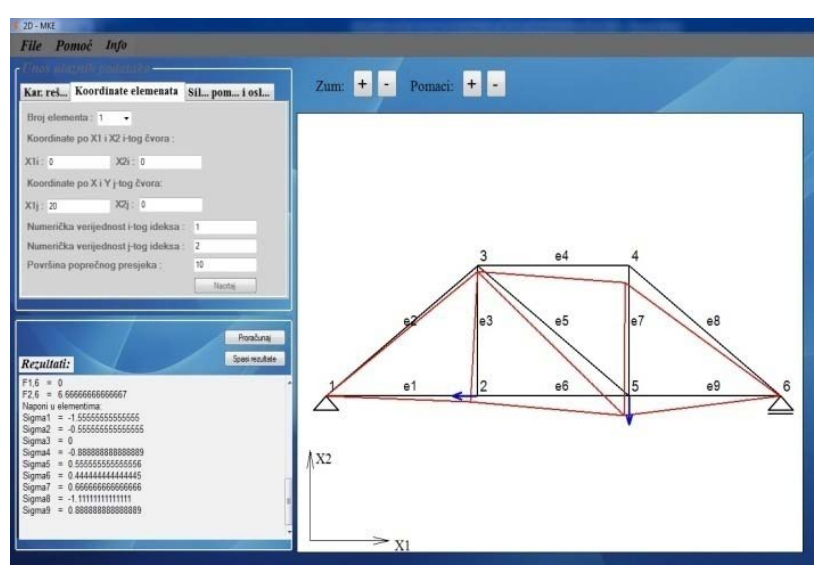

Fig. 1. Graphical interface of the developed software 2D-FEM

In relation to the original software, the developed software 2D-FEM, now has a graphical interface (Fig. 1.) intended for the communication with a user. The graphical interface was designed in the Visual Studio 2008 in C\# programming language. There are several parts of the graphical interface: part for data input, part for presenting of $2 \mathrm{D}$ truss beam in undeformed and deformed condition, part for results print-out, lace with the buttons File, Help, Info, and a part for zooming of $2 \mathrm{D}$ truss beam presentation.

The developed software 2D-FEM encloses a possibility for input data saving, so data must not be entered again during the repeated estimate. There is a possibility for estimate results saving and print out. Software installs and un-installs in a similar way as any other application. The possibilities of the developed software 2D-FEM are demonstrated in the next paragraph of the paper, in the example of deformity and stress condition of the selected 2D truss beams analysis.

\subsection{Software 2D-FEM Possibilities}

Possibilities of the developed software 2D-FEM are demonstrated in the example of deformity and stress condition of the selected 2D truss beams analysis (analytical solutions can be found in Picuga, 1985).

For the selected 2D truss beam presented in the Fig. 2., it is necessary to fix the displacements and forces in the truss joints, as well as stress in the beam elements by the use of the developed software 2D-FEM.

Input data for all elements are: $A=10 \mathrm{~cm}^{2}, E=30$. $10^{3} \mathrm{~Pa}$.

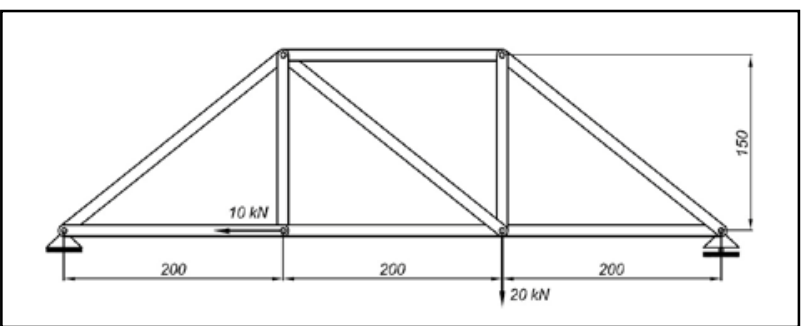

Fig. 2. Presentation of the 2D truss beam in undeformed condition

Before the selected problem solving by the developed software 2D-FEM use, it is necessary, in the same way as we did manually, to mark the truss beam (Picuga, 1985; Cook, 2002).

Solutions obtained by the software 2D-FEM use:

Truss joints displacements [cm]:

$U_{11}=0$

$U_{21}=0$

$U_{14}=-0.00060$
$U_{24}=-0.00215$
$U_{12}=-0.0010$

$U_{22}=-0.00075$

$U_{15}=-0.00074$

$U_{13}=-1.54320 e^{-5}$

$U_{23}=-0.00075$

Forces in the truss joints [kN]:

$F_{11}=20$

$F_{21}=3.3333$

$F_{12}=0$

$F_{22}=0$

$F_{13}=0$

$F_{23}=0$

Stress in the elements $\left[\mathrm{kN} / \mathrm{cm}^{2}\right]$ :

$\sigma_{1}=-1.5555$

$\sigma_{2}=-0.5555$

$\sigma_{3}=0$

$\sigma_{4}=-0.8888$

$\sigma_{5}=0.5555$

$$
\begin{aligned}
U_{25} & =0.002489 \\
U_{16} & =-0.00014 \\
U_{26} & =0 \\
F_{14} & =0 \\
F_{24} & =0 \\
F_{15} & =0 \\
F_{25} & =0 \\
F_{16} & =0 \\
F_{26} & =6.6666 \\
\sigma_{6} & =0.4444 \\
\sigma_{7} & =0.6666 \\
\sigma_{8} & =-1.1111 \\
\sigma_{9} & =0.8888
\end{aligned}
$$

A form of 2D truss beam in deformed condition, presented in the developed software 2D-FEM, is shown in the Fig. 3.

Clearly we are getting results that do not give us the answer abut the way the deformation of 2D truss beams with larger number of elements took place, under the influence of stress. The greatest advantage of the developed software 2D-FEM is in the fact that it has the possibility to visually present the form of the 2D truss beam in deformed condition, based on obtained results which are related to displacements of the truss joins in direction to axis.

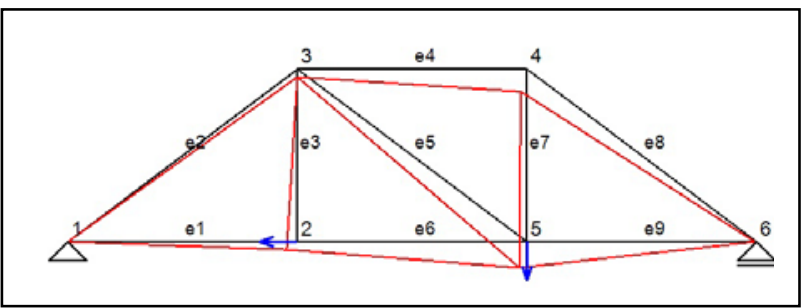

Fig. 3. Presentation of 2D truss beam in deformed condition

\section{CONCLUSION}

We can notice that the use of expensive commercial systems intended for the $2 \mathrm{D}$ truss beams analysis, can be avoided by the use of developed software 2D-FEM. Use of modern programming languages enables use to design visual software which can perform complex calculations. It can also simplify the analysis of the specific structures. In the process of software development it is possible to use principles built into earlier designed console oriented softwares. Although we noticed that the developed software 2D-FEM in current format offers great possibilities for use, there is a need for its further development in order to enable the analysis of deformity and stress condition of 3D truss beams with jointly or firmly tied elements. The use is much more frequent in practice.

\section{REFERENCES}

Budynas-Nisbett (2006). Mechanical Engineering - Shigley’s Mechanical Engineering Design, ${ }^{\text {th }}$ ed., The McGraw-Hill Companies, Inc., ISBN 0-390-76487-6, United States of America

Cook, R.D. at al. (2002). Concepts and applications of finite element analysis, $4^{\text {th }}$ ed., John Wiley and Sons, Inc., ISBN 0-471-35605-0, University of Wisconsin-Madison

Picuga, A. (1985). Introduction to Finite Element Method, Svjetlost, Sarajevo

*** (2011) http://msdn.microsoft.com/en-us/vcsharp/ - Visual C\# Developer Center, Accessed on: 2011-04-18

*** (2011) http://www.csharp-station.com/Tutorial.aspx - C\# Station: C\# Tutorial - Introduction, Accessed on: 2011-0418 